Available online at www.sciencedirect.com

www.cya.unam.mx/index.php/cya

Contaduría y Administración 60 (S2) 83-105

ontaduría y

dministración

\title{
Cointegración entre las principales bolsas de Europa continental en presencia de rompimientos estructurales (1999-2014)
}

\author{
European stock markets' cointegration in the presence \\ of structural breaks (1999-2014)
}

\section{Roberto Joaquín Santillán Salgado ${ }^{\text {a, }}$, Luis Jacob Escobar Saldivara César Gurrola Ríos ${ }^{\mathrm{b}}$}

\author{
aITESM, Campus Monterrey, México \\ ${ }^{b}$ Universidad Juárez del Estado de Durango, México
}

Recibido el 22 de septiembre de 2014; aceptado el 19 de junio de 2015

Disponible en internet el 20 de noviembre de 2015

\section{Resumen}

El objetivo principal de este trabajo es determinar si existe una relación de convergencia de largo plazo entre los cuatro mayores mercados bursátiles de Europa continental y evaluar, al mismo tiempo, el impacto que la volatilidad en uno de ellos tiene sobre el resto. La muestra incluye los mercados de París, Frankfurt, Milán, y Madrid, durante un periodo de importantes cambios en el entorno económico y, en particular, episodios de intensa turbulencia. El enfoque metodológico consiste en la construcción de un modelo capaz de representar los índices bursátiles de los cuatro mercados, para caracterizar su comportamiento histórico mediante técnicas econométricas. El estudio parte de la confirmación de

\footnotetext{
* Autor para correspondencia.

Correo electrónico: roberto.santillan@itesm.mx (R. J. Santillán Salgado).

La revisión por pares es responsabilidad de la Universidad Nacional Autónoma de México.

http://dx.doi.org/10.1016/j.cya.2015.08.013

0186-1042/Derechos Reservados (C) 2015 Universidad Nacional Autónoma de México, Facultad de Contaduría y Administración. Este es un artículo de acceso abierto distribuido bajo los términos de la Licencia Creative Commons CC BY-NC-ND 4.0.
} 
que el comportamiento en el tiempo de los índices bursátiles estudiados no es estacionario en presencia de rupturas estructurales. Validada la evidencia de raíces unitarias, se lleva a cabo un análisis de cointegración de los logaritmos naturales de los índices estudiados y se demuestra la existencia de, al menos, un vector cointegrante. En seguida, se procedió a modelar el comportamiento de las series a través de un modelo de Vectores de Corrección de Errores (VECM), cuyos resultados presentaron problemas de heteroscedasticidad en los residuales, por lo que se recurrió a modelos de la familia GARCH para capturar la complejidad de los factores que determinan ese comportamiento. Efectivamente, una vez incorporado el fenómeno de heteroscedasticidad en el modelado, es posible pasar a la interpretación de los coeficientes del modelo y de la relación compartida de largo plazo entre las series.

Derechos Reservados@2015 Universidad Nacional Autónoma de México, Facultad de Contaduría y Administración.

Este es un artículo de acceso abierto distribuido bajo los términos de la Licencia Creative Commons CC BY-NC-ND 4.0.

Palabras Clave: Integración de mercados de capital; Unión Europea; Cointegración en presencia de rupturas estructurales

\begin{abstract}
The main objective of this work is to determine if there is a long-term convergence relationship among the four largest markets in continental Europe. The sample includes the markets of Paris, Frankfurt, Milan and Madrid during a period of important changes in the economic environment and, in particular, episodes of intense turbulence. The methodological approach used consists in the construction of a model capable of representing the levels of the stock markets of the four markets and of characterizing their historical behavior with the use of econometric techniques. The study begins with the confirmation that the behavior thorough time of the stock market indices studied is not stationary in the presence of structural breaks. After confirming the evidence of unitary roots, a cointegration analysis of the natural logarithms of the indices is performed, confirming the existence of, at least, one cointegrating vector. Next, the behavior of the series was modeled with a Vector Errors Correction Model (VECM). The results of the model presented heteroscedasticity problems, so a GARCH family model was used to capture the complexity of the factors that determine that behavior. In effect, once heteroscedasticity was modeled, it is possible to proceed with the interpretation of the coefficients of the model, as well as the nature of the long-term relationship among the series.

All Rights Reserved (C) 2015 Universidad Nacional Autónoma de México, Facultad de Contaduría y Administración.

This is an open access item distributed under the Creative Commons CC License BY-NCND 4.0.
\end{abstract}

Keywords: Equity markets integration; European Union; Cointegration in presence of structural breaks 


\section{Introducción}

La convergencia de los mercados de capital a nivel internacional es un fenómeno resultante de la influencia de múltiples factores económicos, políticos y tecnológicos. Cabe resaltar el papel correspondiente a los avances en el ámbito tecnológico, los cuales han hecho posible mejorar la velocidad y confiabilidad de las comunicaciones, del procesamiento y almacenamiento de datos, etcétera, así como el significativo rol que han desempeñado los procesos de transformación de la regulación nacional e internacional para hacerse más acorde a las fuerzas económicas y a los procesos de globalización en todas las industrias. En ese contexto, la adopción del euro como moneda única por parte de las mayores economías europeas en 1999 fue un paso en una dirección hasta entonces inexplorada, y buscó potenciar las sinergias naturales de las economías de los países participantes al permitir un flujo libre de capital, trabajo y mercancías.

Durante los años posteriores a la adopción del euro, el sistema financiero ha representado un catalizador relevante para la modernización de la economía de la eurozona. También es importante considerar el impacto favorable que representa el ser una economía impulsada por mayores escalas de operación, beneficiada por una diversificación de riesgos de mercado, y en la cual se propicia la división internacional del trabajo en un ámbito exento de restricciones para el comercio transfronterizo, sobre el desarrollo de los mercados financieros. Indudablemente, en Europa, éstos han experimentado cambios cuantitativos y cualitativos de la mayor trascendencia durante los últimos quince años. De ahí el interés por conocer mejor esa nueva dinámica.

El objetivo de este estudio consiste en investigar si existe una relación de convergencia de largo plazo entre los cuatro principales mercados de capital en Europa continental y evaluar al mismo tiempo el impacto que la volatilidad en uno de ellos tiene sobre el resto. Los mercados estudiados incluyen el alemán, cuyo comportamiento se refleja en el índice DAX; el francés, en el CAC 40; el italiano, en el índice MIB, y el español, en el IBEX. La elección responde al tamaño de las economías nacionales correspondientes, así como a la profundidad y amplitud de sus mercados de capitales.

La formación de la Unión Europea — resultado de la evolución de instituciones internacionales originadas por los países de la región, con el propósito de promover el comercio y la inversión transfronterizos — creó condiciones altamente favorables para que los mercados de capital correspondientes desarrollaran entre sí importantes similitudes. En este trabajo se aborda, desde una perspectiva econométrica, el análisis de dicha convergencia, así como de los efectos de numerosos 
episodios de volatilidad de los mercados provocados por circunstancias diversas durante los últimos quince años.

El enfoque metodológico elegido propone la construcción de un modelo capaz de representar los rendimientos de los índices bursátiles de los cuatro mercados estudiados con base en modelos que caracterizan su comportamiento histórico y sus interrelaciones.

El estudio parte de la confirmación de que los índices bursátiles de los cuatro mercados son series de tiempo no estacionarias en niveles durante el periodo analizado, aun en presencia de rompimientos estructurales tan importantes como la burbuja de las empresas de la Nueva Economía, la incertidumbre ocasionada por la guerra de Irak en 2003, la gran recesión de 2007-2009 y la crisis de la deuda soberana de 2010-2012.

Corroborada la existencia de raíces unitarias en las series, se lleva a cabo un análisis de cointegración de los índices, en presencia de rompimientos estructurales. Se utiliza la técnica de análisis de cointegración desarrollada para series de tiempo afectadas por dicho fenómeno (Johansen, Mosconi y Nielsen, 2000). Los resultados del modelado indicaron la presencia de al menos una relación cointegrante entre las cuatro series. En seguida, se procedió a modelar el comportamiento de los índices originales mediante la metodología de Vectores de Corrección de Errores (VECM). Los resultados del VECM confirmaron la presencia de relaciones de equilibrio de largo plazo entre los mercados de capital eurpeos; no obstante, se revelaron señales de falta de consistencia con el supuesto de que la matriz de varianzas y covarianzas es constante en el tiempo. Por lo anterior, así como por la presencia generalizada de colas pesadas en las series, se procedió a incluir los vectores de corrección del error, obtenidos del modelo VECM en un modelo GARCH multivariado. Se utilizó para dicho propósito la especificación VECM-GARCH (Brooks y Persand, 2002; Anoruo y Braha, 2008). Efectivamente, una vez modelada la heteroscedasticidad, fue posible pasar a la interpretación de los coeficientes de las cuatro ecuaciones de la media, de las varianzas y de las covarianzas condicionales.

En la siguiente sección se lleva a cabo una revisión de la literatura sobre integración de mercados bursátiles y se enfatiza el análisis de aquellos estudios cuyo enfoque se centra en los mercados europeos. En la tercera sección se presentan los detalles del modelado econométrico; en la cuarta sección, se consignan los resultados obtenidos, así como su interpretación. La última sección corresponde a las conclusiones del estudio y sugiere nuevas líneas de investigación. 


\section{Revisión de la literatura sobre integración internacional de mercados de capitales}

Desde la perspectiva de los inversionistas, uno de los resultados más notables de la globalización sobre la industria financiera ha sido la creciente sensibilidad de los mercados de capitales domésticos a los acontecimientos ocurridos en mercados extranjeros. Se habla de «mercados de capital integrados», en el sentido de que efectos de corto plazo de un evento surgido en algún país se transmiten a otros mercados y, en el largo plazo, comparten tendencias comunes (López, Ortiz y Cabello, 2009).

En ese sentido, es posible argumentar que eventos inesperados, como las crisis financieras de los últimos 20 años, han propiciado una mayor integración financiera. Así, por ejemplo, Climent y Meneu (2000), al estudiar cómo la crisis asiática de 1997 afectó las relaciones entre los mercados bursátiles internacionales, comentan que no se detectó la presencia de relaciones de equilibrio multivariantes de largo plazo, pero que el rol del mercado americano como líder del resto del mundo se reforzó tras el periodo de la crisis y la respuesta de los mercados asiáticos ante shocks externos se exacerbó. También, describen una profundización en la integración de los mercados asiáticos con el resto de los mercados internacionales y, por último, reportan que el efecto contagio se manifestó en las relaciones dinámicas entre los mercados bursátiles internacionales.

Licht (1997) utiliza el término «integración de mercados accionarios» en el sentido más amplio, incluyendo le presencia de fenómenos tales como el listado de títulos en varios mercados simultáneamente, las transacciones bursátiles transfronterizas y la provisión de servicios de inversión por parte de empresas extranjeras en el mercado doméstico. Estudia la evolución de los principales mercados europeos desde el punto de vista de su operación cotidiana, su forma de organizarse, su tamaño relativo, etcétera; pero también aborda el análisis del sistema de listado de ciertos títulos en múltiples mercados, el tema de transacciones transfronterizas y las operaciones de arbitraje a través de las fronteras.

La creación de mecanismos formales para la creación del mercado accionario integrado en Europa, incluido el Eurolist, que permite a las compañías basadas en un país miembro obtener el listado de sus acciones en otros países europeos utilizando la misma documentación, así como otras circunstancias diversas que han promovido un creciente volumen de operación de acciones y bonos de otros países en las principales bolsas, han contribuido a la creciente integración de los mercados de capital europeos.

Todo lo anterior, en un contexto caracterizado por el surgimiento y adopción generalizada de las nuevas tecnologías de comunicación y procesamiento de datos, 
la introducción de nuevos sistemas de cotización (Euroquote, Nordquote, etcétera) y la favorable disposición de los países para contribuir a un mercado unificado funcionalmente, aunque aún segmentado desde un punto de vista institucional, habían dado lugar ya, en 1997, a un crecimiento muy importante de los mercados europeos. Éstos y muchos otros abundantes detalles institucionales contenidos en el trabajo Licht (1997) lo hacen una referencia indispensable para el estudio de los procesos integración de los mercados bursátiles en Europa.

En la literatura financiera, la expresión «integración de mercados» también se utiliza frecuentemente para describir una situación en la cual los activos financieros convergen hacia un mismo perfil de riesgo y rendimiento y, por lo tanto, observan el mismo precio una vez que se toma en consideración el efecto del tipo de cambio (cuando se trata de países con distintas monedas). Sin embargo, las dos acepciones anteriores no son excluyentes y, en realidad, están estrechamente relacionadas debido a que tanto el listado de títulos en varios mercados como las transacciones internacionales conducen a la «integración», en el primer sentido que se mencionó.

Otro autor que se refiere a la integración europea, Rouwenhorst (1999), plantea que existe una creencia generalizada de que los efectos «país» observados en algunas regiones del planeta tienden a desaparecer en Europa Occidental a medida que adopta una moneda única para la región. El argumento se desarrolla a partir de la creciente coordinación entre las políticas monetaria y fiscal de los países de la eurozona. Durante el periodo 1994-1999, el diferencial entre tasas de interés para los países de la unión monetaria europea disminuyó, y los gobiernos comenzaron a realizar esfuerzos importantes para ajustar sus déficits fiscales, en apego a los lineamientos establecidos en el tratado de Maastricht de 1992. Aunque en aquel momento la posibilidad de la desaparición de diferencias específicas en los rendimientos de los mercados accionarios entre los países de la eurozona era solo un tema especulativo, la historia reciente del comportamiento de los mercados europeos posteriores a la firma del tratado de Maastricht es más representativa de lo que podría esperarse.

En su trabajo, Rouwenhorst documentó que a pesar de la coordinación fiscal y monetaria que ha existido en la eurozona a partir de 1992, los efectos «país» no habían tendido a desaparecer sino, por el contrario, representaron el mayor peso específico en la explicación del comportamiento de acciones individuales para el periodo analizado, 1978 -1998.

En contraste con los resultados anteriores, Rangvid (2001) utilizó la base de datos de las Estadísticas Financieras Internacionales del Fondo Monetario Internacional para obtener información sobre los índices de precios accionarios trimestrales de 
Francia, Alemania y Reino Unido, entre el primer trimestre de 1960 y el primer trimestre de 1999, y llevar a cabo un análisis VAR con dos rezagos con el propósito de probar la hipótesis de una creciente convergencia entre dichos mercados.

De forma previa, el autor había comprobado que las series utilizadas no eran estacionarias, por lo que procedió a realizar la prueba correspondiente mediante la estimación del número de vectores cointegrantes significativos en distintos puntos del tiempo. La prueba consistió en observar si dicho número aumentó a medida que el periodo de la muestra se extendía. Específicamente, si el número de vectores cointegrantes aumenta a medida que el periodo de la muestra se extiende, no puede descartarse la hipótesis de que las series están vinculadas en forma incremental (por las relaciones cointegrantes) y, por lo tanto, influidas cada vez más por los mismos shocks.

El análisis de Rangvid (2001) concluyó que los tres índices accionarios comparten tendencias estocásticas comunes, al evaluarse para el periodo completo, en tanto que las pruebas recurrentes indican un aumento en el número de vectores cointegrantes a medida que el periodo muestral se amplía. Además, el comportamiento de los índices de precios parece ser conducido por shocks con efectos permanentes, desde el comienzo de la década de 1980. Se concluye que los tres principales mercados accionarios europeos son afectados crecientemente por los mismos factores económicos.

Para Ansotegui y Esteban (2004) la integración financiera se presenta en términos de los determinantes de los rendimientos de las acciones; es decir, la integración del mercado europeo es interpretada como la pérdida de relevancia explicativa del factor «país» en la determinación de los rendimientos accionarios; en cambio, adquiere una mayor influencia el factor «sector». Argumentan que, con la introducción del euro, tuvo lugar una importante reorganización de los inversionistas institucionales internacionales, quienes optaron por cerrar oficinas en varios países europeos y centralizar todas sus operaciones y procesos de decisión por sectores.

El cambio en la forma de operar el proceso de toma de decisiones de inversión, pasando de la diversificación por «país» a por «sector», puede interpretarse como la culminación del proceso de integración de los mercados accionarios europeos. En un mercado integrado por completo, la ubicación geográfica de las empresas emisoras deja de ser relevante, y la correlación entre activos pasa a ser determinada por factores internacionales, en lugar de nacionales. El trabajo de Ansotegui y Esteban utiliza la metodología propuesta por Heston y Rouwenhorst (1995), cuyo postulado es que el rendimiento de una acción en un periodo determinado es explicado por dos factores: la industria a la cual pertenece la empresa y el país de ubicación. Al correr una regresión lineal simple, en la cual el rendimiento de la acción 
individual es la variable dependiente y dicho rendimiento se expresa en función de una constante común para todas las acciones, más una variable que identifica a los distintos sectores y otra que identifica a los distintos países, un mercado accionario está «integrado» del todo cuando el componente país deja de ser estadísticamente significativo, y «no integrado» cuando el coeficiente correspondiente al sector de actividad deja de ser significativo.

Los resultados de Ansotegui y Esteban (2004) sugieren que el factor «sector» es una variable explicativa mucho más significativa después de 1999. No obstante, el factor «país» continúa jugando un rol importante en la explicación de los rendimientos y, argumentan los autores, no puede ser ignorado. El trabajo concluye que, aunque en efecto el euro ha contribuido a aumentar la integración entre los mercados de acciones europeos, ésta aún no se ha logrado de forma total.

Aggarwal, Lucey y Muckley (2004) examinaron la integración de los mercados accionarios europeos para el periodo 1985-2002 mediante tres técnicas econométricas complementarias. En primer lugar, utilizaron un análisis de cointegración tradicional; en un segundo momento, emplearon la técnica basada en el filtro de Kalman, desarrollada por Haldane y Hall (1991) y, por último, efectuaron un análisis de cointegración dinámica mediante la estimación recurrente de eigen-valores, gracias a la cual es posible interpretar con mayor claridad el comportamiento de las correlaciones multilaterales en el tiempo. Las tres técnicas arrojaron resultados equivalentes en el sentido de corroborar que durante los años recientes había tenido lugar un creciente nivel de integración entre los mercados de capital europeos. Pero el incremento de los niveles de integración, en especial durante 1997-1998, cuando se observaron niveles de integración significativamente superiores, conduce a una interesante conclusión en el sentido de que, a pesar de los muchos años de actividad política y demostraciones de voluntad de parte de los líderes europeos para impulsar el proyecto de la Unión Europea, los mercados financieros reconocieron la integración europea efectivamente hasta el establecimiento de la unión monetaria y la creación del Banco Central Europeo.

Kim, Moshirian y Wu (2006) examinaron la influencia de la unión monetaria europea sobre la dinámica del proceso de integración de los mercados accionarios de los países miembros durante el periodo del 2 enero 1989 al 29 mayo 2003. Los autores utilizaron una metodología EGARCH multivariada con correlaciones cambiantes en el tiempo y encontraron que, en efecto, es posible identificar con claridad un cambio de régimen en el proceso de integración de los mercados de la región a partir de la introducción del euro.

Estos autores no fueron los primeros en ofrecer alguna evidencia del impacto de la introducción del euro sobre la integración de los mercados accionarios de Eu- 
ropa (ver, por ejemplo, Hardouvelis et al., 2006; Fratzcscher, 2002). Sin embargo, los estudios previos fueron algo limitados en su cobertura temporal y en el número de países miembros de la unión monetaria europea que fueron incluidos. La contribución central del trabajo de Kim, Moshirian y Wu (2006) consiste en haber obtenido evidencia comprensiva sobre la evolución de los mercados accionarios a nivel regional y global durante el periodo posterior a la introducción del euro y hasta antes de la ampliación de la unión monetaria de 12 a 15 miembros.

Los autores concluyen que: a) tuvo lugar un cambio de régimen entre los mercados accionarios de los países originales de la unión monetaria europea, el cual apunta a una profundización de los canales de transmisión de la información que afectan los rendimientos; b) la unión monetaria causó una mayor integración entre los mercados de los estados miembros y con respecto a Japón y EE.UU.; c) la integración de los mercados accionarios es, en primera instancia, un proceso persistente y estacional, en el cual el desarrollo del mercado accionario es vital; d) el efecto «enero» es significativo, pero contrario a lo reportado por Karolyi y Stulz (2003) sobre la simultaneidad de rendimientos entre el mercado japonés y el de EE.UU.; e) aunque la unión monetaria promovió la integración de los mercados accionarios, la reducción de la volatilidad de los tipos de cambio entre monedas fue importante solo para un pequeño grupo de países, cuya estructura económica es históricamente distinta, y la convergencia económica dentro de la región ha tenido impactos diferenciados sobre la integración de los mercados accionarios.

Mylonidis y Kollias (2010) evalúan el proceso de convergencia dinámica entre los cuatro mayores mercados accionarios europeos, durante la primera década después de la introducción del euro. Su trabajo investiga y compara las tendencias de convergencia en los rendimientos obtenidos en los mercados europeos durante el periodo. Evalúa en qué medida están integrados los principales mercados accionarios de la eurozona y, en forma destacada, si el nivel de integración se ha intensificado a medida que ha transcurrido el tiempo desde la introducción de la moneda única en Europa en 1999 cuando, como sería de esperarse, el proceso de integración económica entre los países miembros se profundizó. Los mercados accionarios estudiados fueron los de Alemania, Francia, España e Italia, los mayores y más desarrollados mercados en la eurozona, los cuales también corresponden a las cuatro mayores economías de la región en términos de PIB y son, al mismo tiempo, la columna vertebral de la planta industrial de la economía de Europa.

El análisis de los niveles de convergencia observados entre los países de la eurozona a partir de la adopción del euro tiene una ventaja significativa sobre el estudio de los mismos mercados accionarios, pero aún denominados en diferentes monedas nacionales antes de la introducción de la moneda única, ya que permite 
un análisis de la convergencia de los rendimientos accionarios en el tiempo, sin necesidad de llevar a cabo ajustes para reconocer las dificultades que surgen en la interpretación empírica cuando existen «premios por riesgo» específicos para distintas monedas nacionales.

Mylonidis y Kollias (2010) argumentan que es importante tomar en cuenta explícitamente las variaciones de las relaciones entre mercados accionarios en el tiempo. Por lo tanto, analizan dichas relaciones utilizando pruebas que reconocen los posibles rompimientos estructurales endógenos presentes en las series. La cointegración recurrente, a su vez, permite obtener una imagen más clara de la posible dinámica de los vínculos entre los mercados accionarios europeos, puesto que, aunque el tamaño de la muestra se mantiene constante, el periodo de observaciones se desplaza hacia adelante una observación a la vez. Adicionalmente, el análisis de cointegración recurrente es muy útil para registrar los múltiples cambios estructurales en las interrelaciones subyacentes y, por lo tanto, para evaluar el proceso de convergencia en términos de grado y de temporalidad.

\section{Metodología}

En la literatura financiera se reconoce que el grado de integración financiera entre los mercados aumenta cuando algún evento de corto plazo en uno de ellos se transmite al resto, y surgen de esa manera las relaciones de largo plazo derivadas de tendencias comunes. Así, Kasa (1992) sugiere que, al estudiar la relación de rendimientos en mercados de capitales, en donde se presume la existencia de tendencias comunes, se incluyan tales relaciones mediante los términos de corrección al equilibrio, como es el caso en los modelos vectoriales de corrección de errores (VECM), ya que el grado de integración entre los mercados se puede capturar a partir del número de relaciones cointegrantes entre ellos; es decir, para el mayor nivel de integración entre $k$ mercados sería de esperar encontrar $k-1$ relaciones cointegrantes, mientras que, en el caso de integración parcial, el número esperado sería menor; finalmente, si los mercados están segmentados del todo, no se encontraría ninguna relación de cointegración.

El modelo $\mathrm{GARCH}^{1}$, desarrollado de manera independiente por Bollerslev (1986) y Taylor (1986), es una generalización multivariada de los modelos autorregresivos condicionalmente heterocedásticos $(\mathrm{ARCH})$ los cuales, en términos de Engle (1982), suponen que la varianza depende de las noticias o shocks pasados. De forma tradicional, los modelos GARCH han sido utilizados para analizar la interrelación de las volatilidades en los mercados financieros, estos modelos asu-

\footnotetext{
${ }^{1}$ Generalized Autoregressive Conditional Heteroscedasticity Model.
} 
men que la varianza condicional depende también de sus propios rezagos, es decir, como una función ponderada del valor promedio $\alpha_{0}$, de la volatilidad del periodo previo $\alpha_{i} u_{t-1}^{2}$, y de la varianza condicional anterior. El modelo queda definido por la ecuación siguiente:

$$
h_{i t}=\alpha_{0}+\sum_{i=1}^{q} \alpha_{i} u_{t-1}^{2}+\sum_{j=1}^{p} \beta_{j} h_{t-j}+u_{t}
$$

Las relaciones modeladas bajo esta especificación difieren de la forma lineal clásica, en donde los parámetros son calculados sobre la base de la ecuación de la media y no de la varianza; así pues, bajo la óptica de los modelos GARCH, se utiliza la técnica conocida como Ajuste por Máxima Verosimilitud, la cual encuentra los valores más probables de los parámetros para la especificación modelada. A continuación, se presenta la ecuación de Máxima Verosimilitud a maximizar:

$$
L=-\frac{T}{S} \log (2 \pi)-\frac{1}{2} \sum_{t=1}^{T} \frac{\log \left(\sigma_{t}^{2}\right)-\frac{1}{2} \sum_{t=1}^{T}\left(y_{t}-\mu-\phi y_{t-1}\right)^{2}}{\sigma_{t}^{2}}
$$

Además de modelar la varianza condicional, la especificación multivariada de los modelos GARCH estima el comportamiento condicional de las covarianzas a lo largo del tiempo, de acuerdo con el modelo siguiente:

$$
h_{i, i, t}=\alpha_{i, 0}+\sum_{r=1}^{R} \alpha_{i, i, r} u_{j, t-r}^{2}+\sum_{s=1}^{S} \sum_{j} \beta_{i, j, s} h_{i, j, t-s}+u_{t}
$$

Bajo el supuesto de normalidad condicional, los parámetros de la ecuación anterior pueden ser estimados maximizando una función de verosimilitud distinta, la cual se presenta a continuación:

$$
l(\theta)=-\frac{T N}{2} \log (2 \pi)-\frac{1}{2} \sum_{t=1}^{T}\left(\log \left|H_{t}\right|+\Xi_{t}^{\prime} H_{t}^{-1} \Xi_{t}\right)
$$

Donde $\theta$ representa los parámetros a ser estimados, $N$ se refiere al número de activos considerados en la modelación, $T$ es la cantidad de observaciones, $H_{t}$ es la matriz de varianzas y covarianzas y $\Xi$ es el vector de innovaciones del sistema. Dicho proceso parte del supuesto de normalidad condicional; sin embargo, cuando no se logra tal condición, los parámetros estimados pueden seguir siendo consistentes, siempre y cuando la especificación sea correcta y se utilicen errores estándar ro- 
bustos. A este procedimiento, propuesto por Bollerslev and Wooldridge (1992), se le conoce como Cuasi-máxima verosimilitud (QML).

\section{Resultados de la modelación}

La información utilizada para el análisis econométrico fueron los logaritmos de los precios de cierre diarios de los índices más representativos de los cuatro mayores mercados de capital de Europa continental, a saber: el CAC 40 para Francia, el DAX para Alemania, el MIB para Italia y el IBEX para España. Las series fueron obtenidas del servicio de información financiera Bloomberg, para el periodo que comprende desde el momento de la adopción del euro, el 4 de enero de $1999^{2}$, hasta el día 24 de junio de 2014. Los índices fueron valuados originalmente con base en los precios de cierre expresados en euros (para evitar el problema de conversión de moneda durante los primeros dos años del euro, ya que durante ese periodo se empleaban de forma simultánea las monedas nacionales y el euro para expresar las cotizaciones), y se excluyó días feriados, para totalizar 3970 observaciones útiles.

Durante ese periodo tuvieron lugar al menos dos episodios de influencia generalizada, tanto para los mercados financieros europeos como para los del resto del mundo. El primero de ellos fue el desplome de las acciones relacionadas con las empresas de la llamada «nueva economía» ${ }^{3}$, entre 1999 y finales de 2002, provocando una tendencia bajista que se vio profundizada por la incertidumbre asociada a los ataques terroristas del 11 de septiembre de 2001, en EE.UU. Como puede observarse en la representación gráfica de las series (fig. 1), dicho episodio provocó una inflexión en la tendencia de las series, para iniciar su recuperación a partir de los primeros días de marzo de 2003. El segundo episodio que tuvo repercusiones y efectos de amplitud internacional sobre los mercados bursátiles fue la llamada crisis de las hipotecas de baja calidad. Fue originada durante 2007 en dicho segmento de EE.UU., pero debido a la utilización de diversos tipos de instrumentos financieros respaldados con activos ${ }^{4}$, se difundió rápido al resto del mundo desarrollado y, aunque en menor medida, también se vio reflejada en el comportamiento de varios mercados emergentes. A lo largo de todo 2008, la crisis se manifestó con mucha

\footnotetext{
${ }^{2}$ Aun cuando los países que adoptaron el euro desde un principio, simultáneamente continuaron utilizando su moneda nacional durante tres años más, como periodo de transición necesario, durante el cual todos los precios y cotizaciones se expresaban tanto en euros como en su moneda nacional. A partir del 1 de enero de 1999, se había fijado en definitiva la tasa de conversión permanente que tendrían, con lo cual quedaba excluida la posibilidad de una fluctuación inesperada.

${ }^{3}$ También conocida como la crisis de las empresas dot-com.

${ }^{4}$ Nos referimos a los Asset Backed Securities, entre los cuales, los bonos respaldados por hipotecas de baja calidad comenzaron a provocar incumplimientos en los pagos contractuales como consecuencia de la inflexión de la tendencia en los precios de las propiedades residenciales, de la elevación de las tasas de interés por el Banco de la Reserva Federal y por la desaceleración de la actividad económica en EE.UU.
} 
intensidad, pero el momento de mayor turbulencia tuvo lugar durante los últimos meses de aquel año, inmediatamente después del anuncio de la quiebra de Lehman Brothers, el día 15 de septiembre. Las medidas extraordinarias adoptadas por los bancos centrales y autoridades hacendarias de las mayores economías de Occidente permitieron paliar la gravedad de las consecuencias que habrían de presentarse entre los intermediarios financieros y entre empresas de todos los sectores económicos a pesar de lo cual, la profundidad de la crisis provocó una drástica caída de los mercados bursátiles. Las primeras indicaciones de una recuperación comenzaron a observarse durante los primero días de marzo de 2009. Ambas inflexiones de tendencia sugieren la presencia de rompimientos estructurales en las series que son objeto de este estudio, como se aprecia en la figura 1.

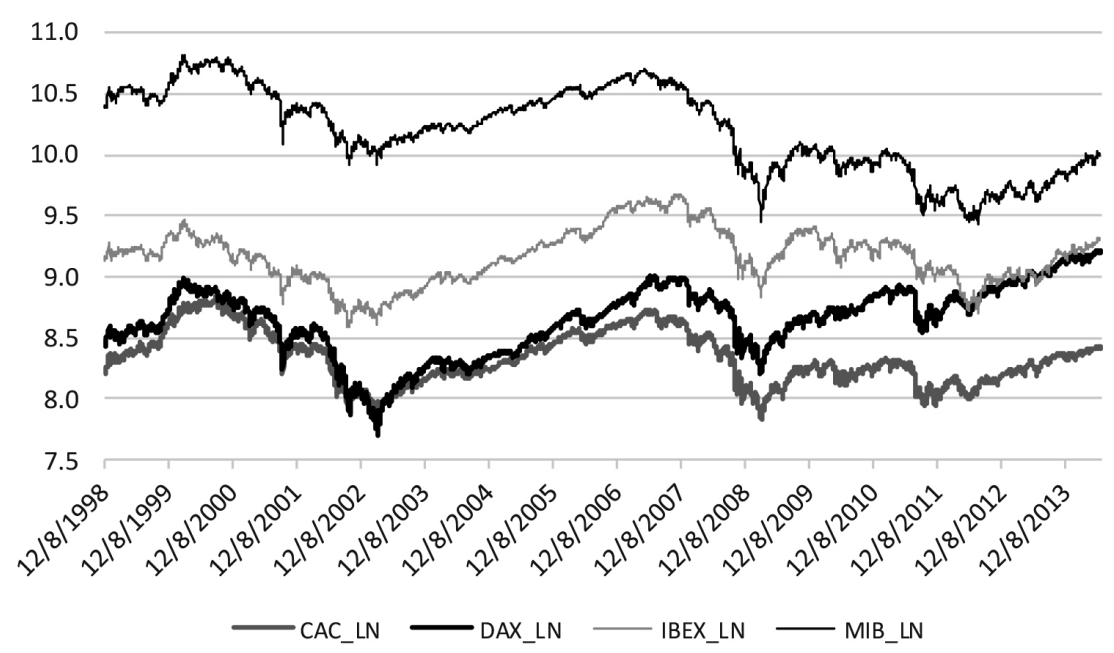

Figura 1. Logaritmos de los precios

El análisis visual de las series sugiere la presencia de rupturas estructurales, por lo que se llevaron a cabo pruebas de raíces unitarias, en las cuales se toma en consideración la posibilidad de que existan, precisamente, rupturas estructurales. Se emplearon las dos pruebas de estacionariedad con rompimiento en las series citadas con más frecuencia en la literatura académica, a saber, la prueba de Perron (1997) y la de Zivot y Andrews (1992)5. Éstas, además, permiten identificar las fechas de los probables rompimientos estructurales y probar la estacionariedad.

En ambos casos, las pruebas contemplan la hipótesis nula de presencia de raíz

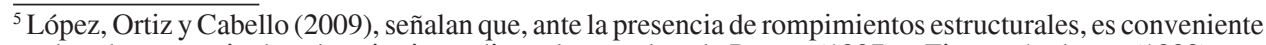
evaluar la presencia de raíz unitaria mediante las pruebas de Perron (1997) y Zivot y Andrews (1992).
} 
unitaria en las series bajo tres posibles variantes de rompimiento estructural: a) rompimiento en el intercepto de la serie con el eje vertical; b) rompimiento en la tendencia de la serie, y c) rompimiento en el intercepto y la tendencia. La tabla 1 presenta los resultados obtenidos con las series estudiadas.

Tabla 1

Pruebas de raíz unitaria con rompimientos estructurales (Datos diarios del 4 de enero de 1999 al 24 de junio 2014).

Panel A: Pruebas de Perron y Zivot y Andrews para detectar la presencia de raíces unitarias en presencia de rompimiento estructural de las series.

\begin{tabular}{|c|c|c|c|c|c|c|c|}
\hline \multicolumn{4}{|c|}{ Prueba Perron (1997) } & \multicolumn{4}{|c|}{ Prueba Zivot y Andrews (1992) } \\
\hline \multicolumn{4}{|c|}{ Valores críticos } & \multicolumn{4}{|c|}{ Valores críticos } \\
\hline Nivel de & \multicolumn{3}{|c|}{ Rompimiento estructural en: } & Nivel de & \multicolumn{3}{|c|}{ Rompimiento estructural en: } \\
\hline Significancia & Intercepto & Tendencia & Ambas & Significancia & Intercepto & Tendencia & Ambas \\
\hline $1 \%$ & -5.92 & -5.45 & -6.32 & $1 \%$ & -5.34 & -4.80 & -5.57 \\
\hline $5 \%$ & -5.23 & -4.83 & -5.59 & $5 \%$ & -4.93 & -4.42 & -5.08 \\
\hline $10 \%$ & -4.92 & -4.48 & -5.29 & $10 \%$ & -4.58 & -4.11 & -4.82 \\
\hline \multicolumn{4}{|c|}{ Valores t } & \multicolumn{4}{|c|}{ Valores t } \\
\hline Índices & \multicolumn{3}{|c|}{ Rompimiento estructural en: } & Índices & \multicolumn{3}{|c|}{ Rompimiento estructural en: } \\
\hline (Logaritmos) & Intercepto & Tendencia & Ambas & (Logaritmos) & Intercepto & Tendencia & Ambas \\
\hline $\mathrm{CAC}$ & -2.74 & -2.18 & -3.07 & $\mathrm{CAC}$ & -2.73 & -2.50 & -3.07 \\
\hline DAX & -3.24 & -2.78 & -3.26 & DAX & -3.24 & -3.19 & -3.26 \\
\hline IBEX & -2.94 & -1.98 & -3.17 & IBEX & -2.95 & -2.07 & -3.15 \\
\hline MIB & -3.17 & -1.97 & -2.92 & MIB & -3.16 & -2.13 & -2.88 \\
\hline
\end{tabular}

Panel B: Fechas de rompimiento estructural de las series, de acuerdo con la prueba de Zivot y Andrews (1992).

\begin{tabular}{ccccc}
\hline Prueba & CAC_LN & DAX_LN & IBEX_LN & MIB_LN \\
\hline Intercepto & $23 / 05 / 01$ & $6 / 6 / 2001$ & $17 / 08 / 04$ & $19 / 05 / 08$ \\
Tendencia & $12 / 7 / 2002$ & $23 / 07 / 02$ & $19 / 06 / 01$ & $22 / 02 / 12$ \\
Ambos & $1 / 10 / 2003$ & $1 / 10 / 2003$ & $13 / 03 / 03$ & $13 / 03 / 03$ \\
\hline
\end{tabular}

Los resultados mostrados en el panel A de la tabla 1 no rechazan en ningún caso la hipótesis nula de presencia de raíz unitaria en los logaritmos de los niveles. Es decir, los valores de la prueba «t» fueron insuficientes para rechazar la hipótesis nula, con un nivel de significancia de $10 \%$ o menos. Por lo tanto, se puede afirmar que las series modeladas pueden ser consideradas como I(1) en sus niveles. En el panel B se presentan las fechas identificadas por la prueba de Zivot y Andrews para los rompimientos estructurales de las series, en cualquiera de sus tres modalidades.

Con base en el argumento de que las series de tiempo que presentan fluctuaciones estacionarias en torno a un punto de ruptura constante se describen mejor 
mediante un proceso de caminata aleatoria — random walk - Johansen, Mosconi y Nielsen (2000), proponen evaluar la presencia de vectores de cointegración mediante una generalización del análisis de modelos autorregresivos vectoriales, sugeridos por Johansen (1988). Por lo mismo, se procedió a la aplicación de la prueba de Johansen, Mosconi y Nielsen $(2000)^{6}$, con la intención de determinar si, en presencia de las rupturas estructurales identificadas mediante las pruebas presentadas en la tabla 1, es posible corroborar la existencia de relaciones de largo plazo entre las bolsas europeas.

De forma similar a las pruebas de Zivot y Andrews y de Perron discutidas en los párrafos anteriores, la prueba JMN contempla tres posibilidades diferentes de rompimiento estructural, por lo que se procedió a verificar la presencia de cointegración entre las series bajo cada una de las hipótesis posibles, a saber: a) rompimento en niveles, intercepto incluido; b) rompimiento en niveles, intercepto y tendencia incluidos, y c) rompimiento en niveles y en tendencia conjuntamente, tendencia e intercepto incluidos. El número de rezagos incluidos en la prueba de la traza se determinó a partir de los criterios de información de Hannan-Quinn y de Schwarz, cuyos resultados para todas las variantes de la prueba indicaron de manera consistente la conveniencia de utilizar dos rezagos ${ }^{7}$.

En la tabla 2, a continuación, se presentan los resultados de la prueba JMN con dos rezagos. Los resultados de la tabla 2 permiten rechazar la hipótesis nula de «cero» relaciones cointegrantes con un nivel de significancia mayor a $1 \%$ en las tres variantes consideradas y, por lo mismo, es posible concluir que la evidencia soporta la afirmación de que existe al menos una relación de cointegración entre las cuatro principales bolsas europeas.

Una vez corroborada la existencia de, por lo menos, una relación cointegrante entre las series de tiempo analizadas, y con el propósito de contar con información más precisa sobre la naturaleza de la relación entre las mismas durante los últimos quince años, incluidos los periodos de elevada turbulencia como fueron aquéllos asociados a la invasión de EE.UU. a Irak y la crisis de las hipotecas de baja calidad, se procedió a la modelación mediante un Modelo Vectorial de Corrección de Errores (VECM).

El VECM expresa los cambios en la variable dependiente como una función lineal tanto de los cambios en las variables explicativas como del término de corrección del error. Éste representa la velocidad de convergencia entre el corto y el largo plazo. Ante la posibilidad de que las innovaciones del proceso no sigan una distribución normal, se utilizó una matriz de errores robusta, como lo sugieren Bollerslev y Woodridge (1992).

\footnotetext{
${ }^{6}$ En lo sucesivo, se abreviará como prueba JMN.

${ }^{7}$ Cabe mencionar que el criterio de Akaike en algunas ocasiones indicó seis o nueve rezagos.
} 
Tabla 2

Pruebas de Cointegración.

\begin{tabular}{cccccc}
\hline \multicolumn{5}{c}{ Rompimento en niveles, intercepto incluido } \\
\hline \multicolumn{5}{c}{ Valores Críticos } & \multicolumn{3}{c}{ Resultados } \\
r0 & $10 \%$ & $5 \%$ & $1 \%$ & LR & p-value \\
\hline 0 & 95.17 & 99.31 & 107.38 & 131.31 & 0.000 \\
1 & 69.19 & 72.79 & 79.86 & 67.26 & 0.140 \\
2 & 47.12 & 50.18 & 56.26 & 42.61 & 0.237 \\
3 & 28.81 & 31.33 & 35.43 & 10.11 & 0.565 \\
4 & 14.17 & 16.21 & 20.48 & 8.57 & 0.477 \\
\multicolumn{5}{c}{ L } \\
\hline \multicolumn{5}{c}{ Vompimiento en niveles, intercepto y tendencia incluidos } \\
\hline r0 & $10 \%$ & $5 \%$ & $1 \%$ & LR & p-value \\
\hline 0 & 107.45 & 112.44 & 122.2 & 137.88 & 0.000 \\
1 & 79.04 & 83.39 & 91.95 & 73.79 & 0.205 \\
2 & 54.54 & 58.23 & 65.57 & 41.99 & 0.537 \\
3 & 33.81 & 36.79 & 42.84 & 19.41 & 0.804 \\
4 & 16.39 & 18.51 & 22.94 & 8.12 & 0.706 \\
\hline Rompimiento en niveles y en tendencia conjuntamente, tendencia e intercepto incluidos \\
\hline \multicolumn{5}{c}{ Valores Críticos } \\
r0 & $10 \%$ & $5 \%$ & $1 \%$ & LR & p-value \\
\hline 0 & 132.82 & 138.26 & 148.86 & 170.82 & 0.000 \\
1 & 99.47 & 104.25 & 113.61 & 99.21 & 0.104 \\
2 & 70.09 & 74.18 & 82.26 & 60.86 & 0.342 \\
3 & 44.49 & 47.84 & 54.55 & 33.57 & 0.518 \\
4 & 22.22 & 24.73 & 29.91 & 10.13 & 0.846 \\
\hline \multicolumn{5}{c}{}
\end{tabular}

No obstante que la modelación del VECM sugiere la presencia de relaciones de equilibrio de largo plazo entre los cuatro principales mercados de capital europeos, es preciso destacar que la dinámica de las series modeladas no se apega al supuesto de que la matriz de varianzas y covarianzas de los rendimientos es constante a través del tiempo. Asimismo, es importante reconocer el efecto de colas pesadas en las series modeladas, así como el derrame potencial de volatilidad. Por lo tanto, para capturar dichas características, se procedió a incluir el vector de corrección del error como ecuación de la media en un modelo GARCH multivariado. Es decir, se utilizó una especificación VECM-GARCH, la cual presupone que el segundo momento de la distribución multivariada cambia a lo largo del tiempo. 
Tabla 3

Modelo estimado ${ }^{8}$ panel A: Ecuación de la media condicional.

\begin{tabular}{ccccccccc}
\hline & \multicolumn{2}{c}{ D(CAC) } & \multicolumn{2}{c}{$\mathrm{D}(\mathrm{DAX})$} & \multicolumn{2}{c}{$\mathrm{D}(\mathrm{IBEX})$} & \multicolumn{2}{c}{$\mathrm{D}(\mathrm{MIB})$} \\
Variable & Coef & Prob. & Coef & Prob. & Coef & Prob. & Coef & Prob. \\
\hline CEq & 0.0213 & $\mathbf{0 . 0 2 9 2}$ & 0.0341 & $\mathbf{0 . 0 0 0 2}$ & 0.0301 & $\mathbf{0 . 0 0 6 3}$ & 0.0371 & $\mathbf{0 . 0 0 0 1}$ \\
Constante & 0.0005 & $\mathbf{0 . 0 2 2 2}$ & 0.0006 & $\mathbf{0 . 0 0 2 0}$ & 0.0005 & $\mathbf{0 . 0 2 8 1}$ & 0.0002 & 0.3185 \\
CAC $_{\mathrm{t}-1}$ & -0.3043 & $\mathbf{0 . 0 0 0 0}$ & -0.1993 & $\mathbf{0 . 0 0 0 1}$ & -0.3016 & $\mathbf{0 . 0 0 0 0}$ & -0.2129 & $\mathbf{0 . 0 0 0 0}$ \\
CAC $_{\mathrm{t}-2}$ & 0.0940 & 0.0659 & 0.1032 & 0.0408 & 0.0910 & 0.0766 & 0.1001 & 0.0328 \\
CAC $_{\mathrm{t}-3}$ & -0.1016 & $\mathbf{0 . 0 3 6 6}$ & -0.0777 & 0.1268 & -0.0998 & $\mathbf{0 . 0 3 5 0}$ & -0.1068 & $\mathbf{0 . 0 2 0 0}$ \\
DAX $_{\mathrm{t}-1}$ & 0.2047 & $\mathbf{0 . 0 0 0 0}$ & 0.1246 & $\mathbf{0 . 0 0 3 3}$ & 0.1430 & $\mathbf{0 . 0 0 0 3}$ & 0.1332 & $\mathbf{0 . 0 0 0 3}$ \\
DAX $_{\mathrm{t}-2}$ & -0.0534 & 0.1588 & -0.0206 & 0.6157 & -0.0249 & 0.5000 & -0.0022 & 0.9509 \\
DAX $_{\mathrm{t}-3}$ & -0.0083 & 0.8146 & -0.0255 & 0.4988 & 0.0157 & 0.6433 & 0.0133 & 0.6829 \\
IBEX $_{\mathrm{t}-1}$ & 0.0544 & 0.1655 & 0.0621 & 0.0836 & 0.1265 & $\mathbf{0 . 0 0 0 8}$ & 0.0749 & 0.0381 \\
IBEX $_{\mathrm{t}-2}$ & -0.0252 & 0.4275 & -0.0189 & 0.5377 & -0.0315 & 0.3271 & -0.0314 & 0.3199 \\
IBEX $_{\mathrm{t}-3}$ & 0.0266 & 0.4051 & 0.0113 & 0.7188 & -0.0088 & 0.7892 & 0.0108 & 0.7252 \\
MIB $_{\mathrm{t}-1}$ & 0.0084 & 0.8219 & -0.0164 & 0.6449 & 0.0162 & 0.6662 & -0.0285 & 0.4281 \\
MIB $_{\mathrm{t}-2}$ & -0.0497 & 0.1749 & -0.0679 & 0.0521 & -0.0651 & 0.0652 & -0.0723 & $\mathbf{0 . 0 3 9 0}$ \\
MIB $_{\mathrm{t}-3}$ & 0.0348 & 0.3364 & 0.0683 & 0.0542 & 0.0552 & 0.1292 & 0.0620 & 0.0931 \\
Dummy-romp-est. & 0.0638 & $\mathbf{0 . 0 0 0 0}$ & 0.0638 & $\mathbf{0 . 0 0 0 0}$ & 0.0638 & $\mathbf{0 . 0 0 0 0}$ & 0.0638 & $\mathbf{0 . 0 0 0 0}$ \\
\hline
\end{tabular}

Panel B: ecuaciones de las varianzas y covarianzas condicionales

\begin{tabular}{|c|c|c|c|c|c|c|c|c|}
\hline Variable & $\mathrm{V}(\mathrm{CAC})$ & prob. & $\mathrm{V}(\mathrm{DAX})$ & prob. & V(IBEX) & prob. & $\mathrm{V}(\mathrm{MIB})$ & prob. \\
\hline$\alpha_{0}$ & 0 & 0.0000 & 0 & 0.0000 & 0 & 0.0000 & 0 & 0.0000 \\
\hline$\alpha_{1}$ & 0.0532 & 0.0000 & 0.0562 & 0.0000 & 0.061 & 0.0000 & 0.0535 & 0.0000 \\
\hline$\beta_{1}$ & 0.9375 & 0.0000 & 0.936 & 0.0000 & 0.9291 & 0.0000 & 0.9391 & 0.0000 \\
\hline & \multicolumn{2}{|c|}{ Covarianzas } & \multicolumn{2}{|r|}{ Coef } & \multicolumn{3}{|c|}{ Prob. } & \\
\hline & \multicolumn{2}{|c|}{$\operatorname{Cov}_{\mathrm{CAC}, \mathrm{DAX}}$} & \multicolumn{2}{|r|}{0.8986} & \multicolumn{3}{|c|}{0.0000} & \\
\hline & \multicolumn{2}{|c|}{$\mathrm{COV}_{\mathrm{CAC}, \mathrm{IBEX}}$} & \multicolumn{2}{|r|}{0.8529} & \multicolumn{3}{|c|}{0.0000} & \\
\hline & \multicolumn{2}{|c|}{$\mathrm{Cov}_{\mathrm{CAC}, \mathrm{MIB}}$} & \multicolumn{2}{|r|}{0.8688} & \multicolumn{3}{|c|}{0.0000} & \\
\hline & \multicolumn{2}{|c|}{$\mathrm{COV}_{\text {DAX,IBEX }}$} & \multicolumn{2}{|r|}{0.8011} & \multicolumn{3}{|c|}{0.0000} & \\
\hline & \multicolumn{2}{|c|}{$\operatorname{Cov}_{\mathrm{DAX}, \mathrm{MIB}}$} & \multicolumn{2}{|r|}{0.8219} & \multicolumn{3}{|c|}{0.0000} & \\
\hline & \multicolumn{2}{|c|}{$\mathrm{Cov}_{\mathrm{IBEX}}$} & & 0.8359 & \multicolumn{3}{|c|}{0.0000} & \\
\hline
\end{tabular}

Nota: los valores de la probabilidad de error de Tipo 1 (rechazar la hipótesis nula cuando es verdadera) en «negrillas» significa que su valor es menor a $5 \%$.

Según Anoruo y Braha (2008) y Brooks y Persand (2002), el primer momento de un modelo VECM-GARCH se representa a partir del vector de corrección de error, mientras que el segundo momento se modela a partir del procedimiento de correlaciones constantes (CCC); así, este modelo considera que las correlaciones condicionales son constantes en el tiempo, con la intención de asegurar que las fluctuaciones en las covarianzas condicionales resultan determinadas por shocks en las varianzas.

\footnotetext{
${ }_{8}^{8}$ Las estimaciones se realizaron con el paquete EViews 8.1, con la opción de matriz robusta. Para maximizar la función de verosimilitud se eligió el algoritmo de Marquardt.
} 
En la ecuación de la media condicional presentada en el panel A de la tabla 3, se aprecia que los vectores de corrección de desequilibrios de corto plazo $(\mathrm{CEq})$ son altamente significativos para las cuatro bolsas. Es decir, la modelación econométrica confirma la relación de equilibrio de largo plazo entre los rendimientos de los mercados bursátiles de Francia, Alemania España e Italia. Asimismo, a partir de dicho resultado, es posible inferir que el ajuste hacia el equilibrio es, en todos los casos, lento. No obstante, el MIB y el DAX se ajustan un poco más rápido, mientras que los más lentos en recuperar el equilibrio de largo plazo son el IBEX y el CAC (último lugar). Otro dato interesante de los resultados del panel A tiene que ver con la importancia de la variable dummy, que representa la fecha de rompimiento estructural en las series modeladas (13 de marzo de 2003). Como se puede apreciar, esa variable presenta la mayor significancia estadística en todos los casos. Aun cuando las pruebas de raíces unitarias (Zivot y Andrews y Perron) inicialmente identificaron dos momentos de ruptura estructural en ambos, el intercepto y la tendencia, a saber: a) el 13 de marzo de 2003, y b) el 1 de octubre de 2003 (panel B de la tabla 1), solo el primero demostró tener significancia estadística.

Resulta interesante destacar que la fecha identificada para el rompimiento de la tendencia y el intercepto como la más significativa para el modelo coincidió con la víspera del inicio de la invasión a Kuwait. Aparentemente, los mercados descontaron con una semana de anticipación dicho evento, por lo que se registró una clara inflexión al alza de las cuatro series a partir del 13 de marzo de 2003. Habría sido lógico descubir que tanto el evento de la guerra del Golfo Pérsico como la crisis de las hipotecas de caja calidad (2007-2009), o incluso la crisis de la deuda soberana (2010-2012), podrían haber tenido impactos significativos en la tendencia e intercepto de las series ${ }^{9}$. Sin embargo, la evidencia econométrica sugiere que el efecto más importante sobre el comportamiento de las bolsas europeas fue, paradójicamente, la recuperación de los precios de las acciones que inció una semana después de la fecha identificada (fig. 1), luego de un periodo de baja significativa a partir de 1999 (reventón de la burbuja de precios de las acciones de la nueva economía, también conocido como la crisis de las dot-com).

\footnotetext{
$\overline{9}$ Todas estas fechas de quiebres importantes en las series se presentan en la figura 1, identificadas con líneas verticales.
} 


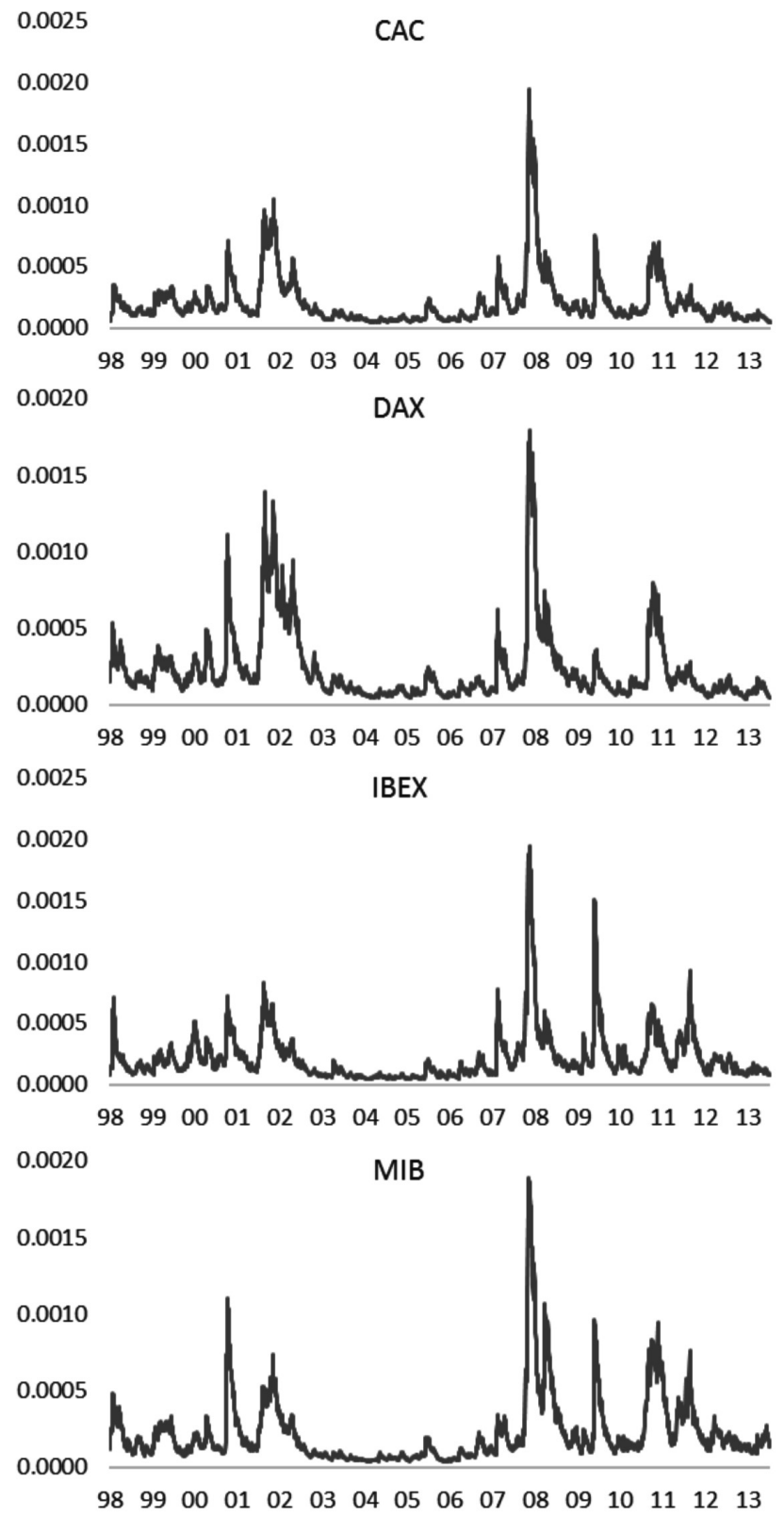

Figura 2. Varianzas Condicionales. 
En el panel B de la tabla 3, se presenta la ecuación de la varianza condicional o volatilidad de los rendimientos de los índices bursátiles europeos, obtenida a partir de los residuales de la ecuación de la media. Los parámetros estimados sugieren que aquella responde a shocks provenientes de su propia volatilidad rezagada (efecto GARCH), así como de los errores rezagados al cuadrado (efecto ARCH). Las cuatro ecuaciones de la varianza condicional revelan procesos estacionarios y persistencia de largo plazo de los shocks de volatilidad, de acuerdo con la evidencia resutlante al sumar los coeficientes asociados a los efectos ARCH y los efectos GARCH en las ecuaciones de los rendimientos de Francia, Alemania, España e Italia: 0.9907, 0.9922, 0.9901 y 0.9926, de forma respectiva. Consecuentemente, la vida media de un choque de volatilidad es de 70 y 74 días para los mercados de España y Francia y de 89 y 93 días en los casos de Alemania e Italia. La figura 2 muestra la dinámica de las varianzas condicionales modeladas con el VECMGARCH.

Como se puede apreciar, la volatilidad condicional de los rendimientos de los mercados de Francia, Alemania, España e Italia durante el periodo estudiado es muy similar. Su respuesta a shocks en prácticamente los mismos periodos, es casi la misma, si bien el grado de respuesta diverge en intensidad.

La integración internacional de los mercados de capital de los cuatro países europeos estudiados es un reflejo de la creciente integración económica, de la liberalización del sistema financiero europeo y de las economías de escala y alcance, obtenidas al lograr mayores niveles de liquidez y profundidad. El proceso resulta en una mejora de las condiciones de financiamiento para las empresas de la zona, al reducir los costos de transacción, permitir el acceso a una mayor variedad de instrumentos de financiamiento y promover mayor eficiencia de los mercados europeos. No obstante, los beneficios de la diversificación logrados mediante la diversificación internacional, en otras circunstancias, se diluyen para los inversionistas europeos que siguen estrategias de largo plazo. La estrategia de inversión de éstos debería incorporar planes de diversificación de portafolios más allá de las fronteras de la zona euro para maximizar sus beneficios y minimizar sus riesgos.

\section{Conclusiones}

La experiencia de integración económica y monetaria de los países miembros de la eurozona es un escenario privilegiado para estudiar las implicaciones de buscar una convergencia económica-financiera intencionada, en contraste con la convergencia que tiene lugar de forma espontánea en otras regiones geográficas. El interés por conocer mejor los procesos y mecanismos que caracterizan a los 
mercados financieros de la eurozona justifica continuar estudiando su realidad y evolución.

Las etapas transcurridas en el proceso de integración dejan constancia de la gradualidad y complejidad de un proyecto de esa magnitud para un conjunto de países, los cuales, por su estructura económica, se ubican en una amplia gama de niveles de desarrollo. El impulso atribuible a los mercados financieros y, en particular, a los mercados de capital, en la modernización y la elevación de la competitividad de las empresas, realza la utilidad de conocer su funcionamiento y sus procesos, tanto desde la perspectiva de los inversionistas locales como de los internacionales.

La mayor profundidad, liquidez y estabilidad, que se logra a medida que hay una mayor participación de inversionistas locales y extranjeros, justifica realizar esfuerzos por conocer mejor la naturaleza del comportamiento y las relaciones entre los distintos mercados locales. Este trabajo representa un paso en esa dirección y se enmarca en una creciente línea de investigación sobre la integración de los mercados de capital europeos en el contexto de la integración económica y financiera de la unión monetaria europea.

El análisis econométrico presentado en este estudio contribuye a la literatura al confirmar la presencia de una relación de largo plazo entre los principales mercados de capital de Europa continental. Aun cuando distintos episodios afectaron la estabilidad de los mercados financieros durante el periodo analizado, al utilizar técnicas robustas a la presencia de rompimientos estructurales, se pudo confirmar la presencia de la cointegración de los cuatro mercados. Existen distinas razones por las cuales podría haberse previsto este resultado, pero solamente la confirmación empírica de esa realidad puede soportar el diseño de estrategias de inversión consistentes con la nueva naturaleza de los mercados de la zona euro.

La utilización de técnicas de modelaje econométrico que permiten discernir la existencia de una relación de largo plazo entre los mercados europeos estudiados es una contribución importante, dada la falta de consenso en la literatura sobre este particular. En cuanto a los problemas econométricos enfrentados a lo largo del estudio, se resolvió el problema de no estacionariedad detectado en las series originales en niveles mediante la utilización de la metodología de cointegración; en seguida, ante la violación de los supuestos de constancia de la matriz de varianzas y covarianzas de los residuales del modelo VECM, se procedió a llevar a cabo un modelaje mediante la técnica VECM-GARCH, poco utilizada hasta ahora en estudios del mercado financiero. Gracias al modelo mencionado, se corrobora la significancia de la relación de cointegración para los cuatro mercados estudiados. El VECM-GARCH corrige los problemas de autocorrelación de los residuales y 
modela la heteroscedasticidad de los mismos, para permitir una interpretación de los parámetros del modelo.

No obstante los resultados discutidos, valdría la pena explorar con mayor profundidad el efecto potencial de colas pesadas, así como profundizar en los mecanismos de transmisión y derrame de volatilidad que afectan tal relación o bien, evaluar el fenómeno con técnicas de orden superior, como la cointegración fraccional. Por el momento diferimos ese esfuerzo para un trabajo futuro.

\section{Referencias}

Aggarwal, R., B. Lucey y Muckley, C., (2004). Dynamics of Equity Market Integration in Europe: Evidence of changes over time and with events. Institute for International Integration, IIIS Discussion Paper No. 19, University of Dublin. Ireland.

Anoruo, E., y Braha, H. (2008). Housing and stock market returns: An application of GARCH enhanced VECM. The IUP Journal of Financial Economics, 6 (2), 30-40.

Ansotegui, Carmen, y Esteban, María Victoria (2004). European Stock Market Integration. ESADE, Barcelona.

Bollerslev, Tim (1986). Generalized Autoregressive Conditional Heteroscedasticity. Journal of Econometrics, (31), 307-327.

Bollerslev, T. y Wooldridge, J.M., (1992). Quasi-Maximum Likelihood Estimation and Inference in Dynamic Models with Time-Varying Covariances. Econometric Reviews, 11 (2), 143-172.

Brooks, C., Henry, O. T., y Persand, G. (2002). The Effect of Asymmetries on Optimal Hedge Ratios. The Journal of Business, 75 (2), 333-352.

Climent, Francisco J. y Meneu, Vicente (2000). ¿Se ha incrementado la transmisión de información entre los Mercados Bursátiles Internacionales a raíz de la Crisis Asiática de 1997? Fundación de Estudios de Economía Aplicada, FEDEA, (82).

Engle, R. F. (1982). Autoregressive conditional heteroscedasticity with estimates of the variance of United Kingdom inflation. Econometrica, (50), 987-1007.

Fratzscher, M. (2002). Financial market integration in Europe: On the effects of the EMU on stock markets. International Journal of Finance and Economics, (7), 165-193.

Hardouvelis, G. A., Malliaropulos, D., y Priestley, R. (2006). EMU and European Stock Market Integration. The Journal of Business, 79 (1), 365-392.

Haldane, A. G. y Hall, S. G. (1991). Sterling's relationship with the Dollar and the Deutschemark: 1976-89. The Economic Journal, (101), 436-443.

Heston, Steven L. y Rouwenhorst, K, Geert, (1995). Industry and Country Effects in International Stock Returns. Journal of Portfolio Management, 3 (21), 53-58.

Johansen, S. (1988), Statistical analysis of cointegration vectors. Journal of Economic Dynamics and Control, (12), 231-254.

Johansen, S., Mosconi, R., y Nielsen, B., (2000). Cointegration analysis in the presence of structural breaks in the deterministic trend. The Econometrics Journal, 216-249.

Karolyi, G. A., y Stulz, R. M. (2003). Are financial assets priced locally or globally? Handbook of the Economics of Finance, 1, 975-1020.

Kasa, K. (1992). Common stochastic trends in international stock markets. Journal of Monetary Economics, 29 (1), 95-124. 
Kim, Suk-Joong; Moshirian, Fari, y Wu, Eliza (2006). Evolution of International Stock and Bond Market Integration: Influence of the European Monetary Union. Journal of Banking and Finance, 5 (30), 1507-1534.

Licht, Amir N. (1997). Stock Market Integration in Europe. Harvard Institute for International Development, CAERII Discussion Paper, (15).

López, Herrera, F., Ortiz, F., E., y Cabello, A., (2009). Las interrelaciones de volatilidad y rendimientos entre los mercados de valores del TLCAN. Investigación Económica, 68 (267), 83-114.

Mylonidis, Nikolaos, y Kollias, Christos. (2010). Dynamic European stock market convergence: Evidence from rolling cointegration analysis in the first euro-decade. Journal of Banking \& Finance, (34), 2056-2064.

Perron, P., (1997). Further Evidence on Breaking Trend Functions in Macroeconomic Variables. Journal of Econometrics, (80), 355-85.

Rangvid, Jespers. (2001). Increasing convergence among European Stock Markets? A recursive common stochastic trends analysis. Economic Letters, 3 (71), 383-389.

Rouwenhorst, K. Geert, (1999). European Equity Markets and the EMU. Financial Analyst Journal, May-June, 57-64.

Santillán-Salgado, R.J., (2015). La Crisis de las Hipotecas de Baja Calidad en Estados Unidos. Capítulo 3, en: La Gran Recesión (2007-2012): Lecciones y Oportunidades para México. Roberto J. Santillán-Salgado, Coordinador. Instituto Mexicano de Ejecutivos de Finanzas y EGADE Business School, México

Taylor, S., (1986). Modelling Financial Time Series,.John Wiley \& Sons, New York.

Zivot, E. y Andrews, D.W.K., (1992). Further Evidence on the Great Crash, the Oil-Price Shock, and the Unit-Root Hypothesis. Journal of Business and Economic Statistics, 10 (3), 251-70. 